\title{
Generalizing the Nagel line to Circumscribed Polygons by Analogy and Constructive Defining
}

\author{
Michael de Villiers \\ School of Science, Mathematics \& Technology Education, University of KwaZulu-Natal \\ profmd@mweb.co.za
}

\begin{abstract}
This paper first discusses the genetic approach and the relevance of the history of mathematics for teaching, reasoning by analogy, and the role of constructive defining in the creation of new mathematical content. It then uses constructive defining to generate a new generalization of the Nagel line of a triangle to polygons circumscribed around a circle, based on an analogy between the Nagel line and the Euler line of a triangle.
\end{abstract}

The purpose of this paper is to heuristically present a new generalisation of the Nagel line of a triangle to polygons circumscribed around a circle by making use of an interesting analogy between the Nagel line and the Euler line of a triangle (De Villiers, 2006).

I hope that by describing the invention process to perhaps contribute in some small way to teachers' perspectives on the nature of mathematics, namely an understanding of how new mathematics is sometimes created. This may also hopefully assist and motivate teachers to attempt to recreate similar learning experiences for their students with content and concepts appropriate to their level.

Therefore, in order to place the invention process in a broader perspective relevant to mathematics education, I will first discuss the genetic approach, reasoning by analogy, and constructive defining, before the generalisation of the Nagel line to circumscribed polygons is presented.

\section{The genetic approach}

Like the mathematician George Polya, I always wondered as a student at high school, and even to some degree at undergraduate level at university, how all the mathematics we were learning - the efficient algorithms, beautiful theorems and sometime ingenious proofs - had been discovered or invented (Polya, 1945). It often seemed to me mysterious and amazing, but sometimes also capricious and intimidating, to be taught theorem after theorem, followed by carefully presented proofs, without been given any insight into how they were found, nor what may have motivated them in the first place. Faced with an unrelenting barrage of mathematics presented in this way, I sometimes felt I had little choice but to succumb to dutiful memorisation and rote learning in order to pass the examinations.

One of the first books that I read that seriously attempted to demystify the origins of mathematics (and that has had a life-long influence on me), was Polya's famous 1945 book How to Solve It, which concentrate on the processes of mathematics, not the end-results or products. This focus on the processes of mathematics is in correspondence with what is often called the 'genetic' approach in mathematics education and was already propounded by the German mathematician Felix Klein (1924):

People often think that mathematics can, or even should be, taught purely deductively, in the sense that a certain set of axioms should serve as a starting point and everything else should be derived logically from it. This approach is often justified on the authority of Euclid, but does not correspond to the historical development of mathematics. ( p. 17)

(The teacher) ought ... to lead pupils slowly along the same path to higher ideas and finally to abstract formulations, as those that the human race in general followed from a naïve primitive state to knowledge at higher, abstract level. (p. 290) 
Likewise, Freudenthal (1973) has criticised a deductive teaching approach, calling it a "didactical inversion" of the historical process, and that it was in fact was anti-didactical. He propagated "mathematics as an activity": students should be given the opportunity to "re-invent" mathematics for themselves. Toeplitz (1963, p. v) eloquently describes the motivation behind the genetic approach and how it may help to demystify mathematics:

Regarding all these basic topics in infinitesimal calculus which we teach today as canonical requisites, e.g., mean-value theorem, Taylor series, the concept of convergence, the definite integral, and the differential quotient itself, the question is never raised "why so?" or "How does one arrive at them?" Yet all these matters must at one time have been goals of an urgent quest, answers to burning questions, at the time, namely, when they were created. If we were to go back to the origins of these ideas, they would lose that dead appearance of cut-anddried facts and instead take on fresh and vibrant life again.

However, for Toeplitz it is clearly not about a complete dramatisation of the history of mathematics:

The historian - the mathematical historian as well - must record all that has been, whether good or bad. I, on the contrary, want to select and utilize from mathematical history only the origins of those ideas which came to prove their value ... It is not history for its own sake in which I am interested, but the genesis at its cardinal points, of problems, facts, and proofs. (Toeplitz, 1963, p. v)

With reference to Klein's genetic principle, Hull (1969, p. 29) similarly writes:

It certainly suggests that the pattern of past mathematical discovery should be closely studied in relation to any proposed pattern of individual learning. This does not mean a slavish adherence to the historical order of development in every detail or topic; but it may well mean that the broad general lines along which the race has conducted its creative thought are also the lines along which children can most naturally learn.

It is therefore encouraging that the Revised National Curriculum Statement (RNCS) (DoE,
2002) acknowledges the importance of the history of mathematics by stating that the Mathematics Learning Area should develop "an appreciation for the diverse historical, cultural and social practices of Mathematics" (p. 13, bold added), and later on gives several examples like the following:

Grade 5 Assessment Standards:

Describes and illustrates various ways of writing numbers in different cultures (including local) throughout history. (p. 49)

Grade 8 Assessment Standards:

Describes and illustrates ways of measuring in different cultures throughout history (e.g. determining right angles using knotted string, leading to the Theorem of Pythagoras). (p. 95)

However, as shown above, the RNCS unfortunately seems to restrict the use and importance of the history of mathematics to only the incorporation of a few historical examples here and there. More generally, one can distinguish at least four ways in which the history of mathematics can be utilised by a teacher:

1) a complete presentation of the historical development of a concept, algorithm or theorem

2) an abbreviated presentation of the most significant moments of the historical development in chronological order

3) a presentation which includes no historical material, but is nonetheless based on an analysis of the historical development of the particular concepts, algorithms and theorems (the indirect, genetic method)

4) a presentation which is not based on the actual historical development of the particular concepts, algorithms and theorems, but attempts to simulate with the advantage of hindsight, how they might've been discovered and/or invented through typical mathematical processes or ways of thinking.

In other words, what is suggested in 4) above, is that a detailed analysis of the history of mathematics can lead to the identification of certain general patterns and processes by which mathematical content is discovered and invented, and that these patterns and processes could then be utilized as possible teaching approaches without any direct reference to the history of the particular content being taught. So for example, it is possible to teach Boolean Algebra as described in De Villiers (1986a), not in the actual historical order nor from the context it originally developed, but to instead begin by focussing on the modelling of 
switching circuit problems, and only later on dealing with its axiomatization into a formal mathematical system of axioms, theorems and proofs (as well as its application to other areas such as logic, computer programming, biology, etc.)

An approach in which learners are exposed to or engaged with the typical mathematical processes by which new content in mathematics is generally discovered, invented and organized has been called a "reconstructive" approach by Human (1978:20) as follows:

With this term we want to indicate that content is not directly introduced to pupils (as finished products of mathematical activity), but that the content is newly reconstructed during teaching in a typical mathematical manner by the teacher and/or the pupils. (freely translated from Afrikaans)

The effective implementation of a reconstructive approach therefore presupposes that teachers themselves are well acquainted and proficient in typical mathematical processes. The history of mathematics is of course not the only source by which teachers can gain useful knowledge and insight into the typical mathematical processes by which new mathematics is discovered and created. Valuable insight can also be obtained by reading books on problem solving, problem posing and heuristic reasoning, and perhaps most importantly, from being mathematically active oneself, and reflecting on one's own struggles and triumphs. It is therefore with this latter view in mind that this paper hopes to make a modestly small contribution, and to which we are now proceeding in the next sections.

\section{Reasoning by analogy}

Analogy is a kind of similarity. Reasoning by analogy is often a powerful means of extending or applying mathematical results into other domains. It has often also featured in several major scientific discoveries. For example, by noticing the analogy between the movement of a projectile and the moon around the earth, Newton was ultimately steered in the direction of his famous laws. Similarly, Kekulé is reputed to have developed the idea of a circular molecular structure for benzene from a dream of a snake swallowing its tail. When first Leonardo da Vinci, and then later the Wright brothers, considered propellers, they respectively regarded them as "air screws" or "rotary wings".
Polya (1954, p. 13) defines an analogy between two mathematical systems as follows: "two systems are analogous, if they agree in clearly definable relations of their respective parts." For example, a circle, triangle and square in the plane are respectively analogous to a sphere, tetrahedron and cube in space. In the plane, a circle may be defined as the locus of all points equidistant from a point, but similarly in space, a sphere can be defined as the surface formed by all the points equidistant from a point. A triangle is the most elementary, finite figure in the plane bounded by straight lines, whereas a tetrahedron is the most elementary solid in space bounded by planes. Lastly, congruent line segments, all perpendicular to each other, bound a square in the plane, while congruent square faces, all perpendicular to each other, bound a cube in space.

Sometimes an analogy can be defined explicitly, for example, when the relations are governed by the same rules or axioms. For example, the addition of numbers is analogous to the multiplication of numbers, since both are commutative and associative. In such a case where the analogy is determined by clearly defined rules, we have a duality, as the two operations can be interchanged (as long as only these laws are involved).

For example, this duality between addition and multiplication extends to a fruitful analogy between arithmetic and geometric sequences to produce an interesting dual for the Fibonacci sequence, involving an analogous rule $T_{n} \times T_{n+1}=T_{n+2}$ for producing consecutive terms (see De Villiers, 2000). Just like the Fibonacci sequence, the limit of the quotients of the logarithms of the adjacent terms of this dual sequence is also the golden ratio, i.e.: $\lim _{n \rightarrow \infty} \frac{\log T_{n+1}}{\log T_{n}}=\phi$.

\section{Constructive defining}

... the algorithmically constructive and creative definition ... models new objects out of familiar ones Freudenthal (1973, p. 458)

In mathematics we can distinguish between two different types of defining of concepts, namely, descriptive (a posteriori) and constructive (a priori) defining (compare De Villiers, 1986b; Human, 1978; Krygowska, 1971). However, for the 
purpose of this paper only constructive defining is relevant.

Constructive (a priori) defining takes places when a given definition of a concept is changed through the exclusion, generalization, specialization, replacement or addition of properties to the definition, so that a new concept is constructed in the process (see Figure 1). In other words, a new concept is defined "into being", the further properties of which can then be experimentally or logically explored. The main purpose or function of a priori defining is the production of new knowledge.

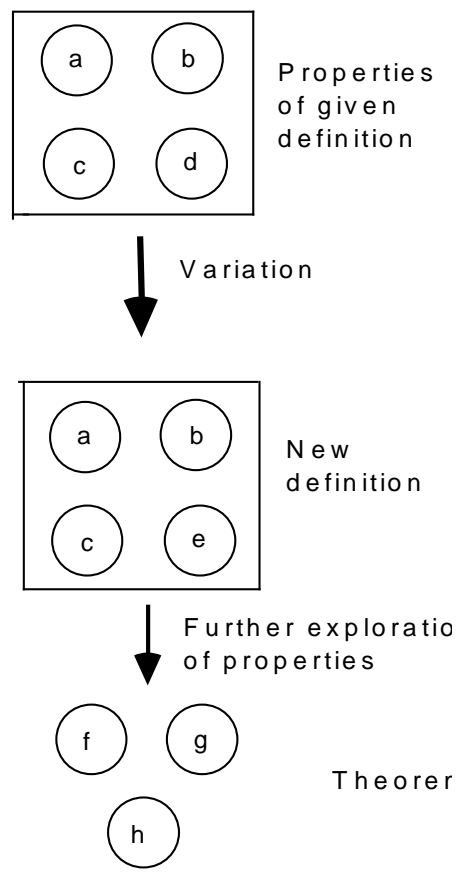

Figure 1: Constructive Defining

A classic, historical example of constructive defining using analogy is the extension of the algebra of the real numbers by Bombelli in 1569 to complex algebra with imaginary numbers, by the addition of an imaginary unit, $i^{2}=-1$, yet maintaining the same basic laws of commutativity, associativity, distributivity, closure and identities. In a letter to his sister, Weil (1940, p. 339) stated: "the analogies between algebraic functions and numbers have been on the minds of all the great number theorists of the time”, and in 1946, he laid the foundations of algebraic geometry from the analogy of the theory of differentiable manifolds with some constructions from algebraic topology.

Another famous example is that of defining a hypercube (a four dimensional cube) by analogy from a three-dimensional cube. Since a cube viewed directly from the front appears like a square within a square with corresponding vertices connected as shown in Figure 2a (i.e. a 2-D representation of a 3-D object), by analogy one can consider a hypercube as a cube inside a cube (i.e. a $3-\mathrm{D}$ representation of a $4-\mathrm{D}$ object) as shown in Figure $2 b$.

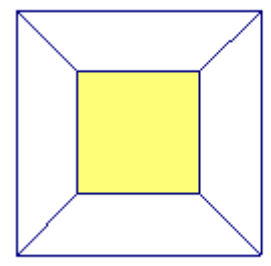

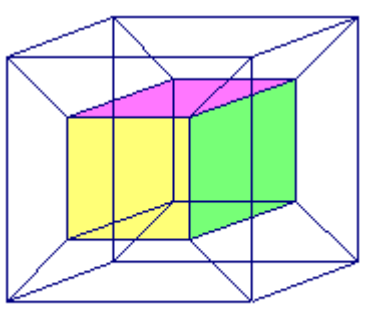

b
Figure 2: Generalising a cube to the fourth dimension

In a similar way, by using analogy we can constructively define a generalisation of the concept of a parallelogram to hexagons by defining a parallelo-hexagon as a hexagon with opposite sides equal and parallel (see Figure 3 ). Or by relaxing one or the other condition, we can generalise even further by constructively defining a parallel-hexagon as a hexagon with opposite sides parallel or a oppo-sided hexagon as a hexagon with opposite sides equal.

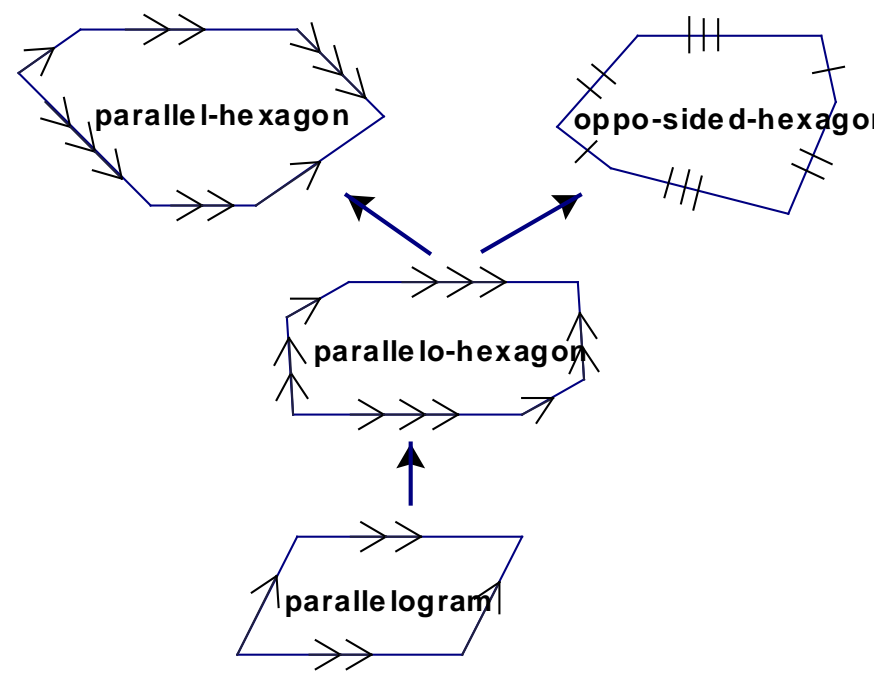

Figure 3: Generalising a parallelogram

\section{The analogy between the Euler and Nagel lines of a triangle}

Analogy seems to have a share in all discoveries, but in some it has the lion's share. (Polya, 1954, p. 17) 
A remarkable analogy between the nine-point circle and Euler line on the one hand, and that of the Spieker circle and Nagel line on the other hand, is contrasted in Table 1 and illustrated in Figure 4 (Coolidge,1971; De Villiers, 2006; Honsberger, 1995).

\section{Generalising the Euler and Nagel lines to quadrilaterals}

Let us now first consider generalising the Euler line to a cyclic quadrilateral. In general, given any quadrilateral $A B C D$ as shown in Figure 5, the respective centroids $C^{\prime}, D^{\prime}, A^{\prime}$ and $B^{\prime}$ of triangles

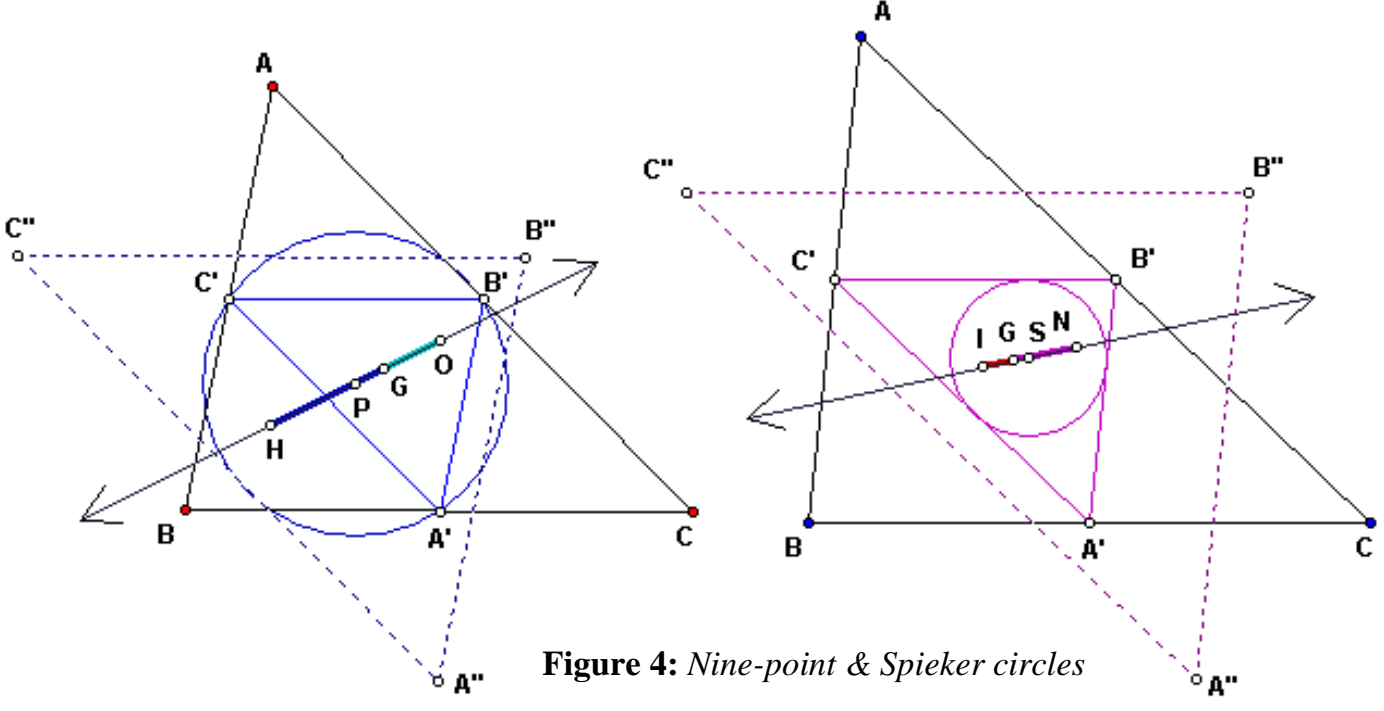

\begin{tabular}{|c|c|}
\hline $\begin{array}{l}\text { The nine-point circle is the circumcircle of } A B C \text { 's } \\
\text { median triangle and has radius half that of } \\
\text { circumcircle of } A B C \text {. }\end{array}$ & $\begin{array}{l}\text { The Spieker circle is the incircle of } A B C \text { 's median } \\
\text { triangle and has radius half that of incircle of } A B C \text {. }\end{array}$ \\
\hline $\begin{array}{l}\text { The circumcentre }(O) \text {, centroid }(G) \text { \& orthocentre } \\
(H) \text { of any triangle } A B C \text { are collinear (Euler line), } \\
G H=2 G O \text { and the midpoint of } O H \text { is the centre of } \\
\text { the nine-point circle }(P) \text { so that } H P=3 P G \text {. }\end{array}$ & $\begin{array}{l}\text { The incentre }(I) \text {, centroid }(G) \text { \& Nagel point }(N) \text { of } \\
\text { any triangle are collinear (Nagel line), } G N=2 G I \\
\text { and the midpoint of } I N \text { is the centre of the Spieker } \\
\text { circle }(S) \text { so that } N S=3 S G \text {. }\end{array}$ \\
\hline
\end{tabular}

It is insightful to compare the underlying similarity transformations implied by both results. For example, note that for the Euler line, a halfturn with centre $G$ and a scale factor of $1 / 2$, maps $A B C$ onto the median triangle $A B^{\prime} C^{\prime}$, and circumcentre $O$ to $P$. But a dilation with a scale factor of 2 from centre $O$, maps $A B^{\prime} C^{\prime}$ to $A^{\prime \prime} B^{\prime \prime} C^{\prime \prime}$, and $P$ to $H$. Therefore, $H$ (the orthocentre of $A B C$ ) is the circumcentre of $A{ }^{\prime \prime} B{ }^{\prime \prime} C$ ".

Similarly, for the Nagel line, a halfturn with centre $G$ and a scale factor of $1 / 2$, maps $A B C$ onto the median triangle $A^{\prime} B^{\prime} C^{\prime}$, and incentre $I$ to $S$. But a dilation with a scale factor of 2 from centre $O$, maps $A B^{\prime} C^{\prime}$ to $A^{\prime \prime} B^{\prime \prime} C^{\prime \prime}$, and $S$ to $N$. Therefore, $N$ (the Nagel point of $A B C$ ) is the incentre of $A^{\prime \prime} B$ "C ${ }^{\prime \prime}$.
$A B D, A B C, B C D$ and $C D A$ form a quadrilateral $A B^{\prime} C D^{\prime}$, similar to the original and scale factor $1 / 3$ (a halfturn and reduction by $1 / 3$ ), with lines $A A^{\prime}, B B^{\prime}, C C^{\prime}$ and $D D^{\prime}$ concurrent in $G$. Then this point of concurrency $G$ (centre of similarity between $A B C D$ and $A^{\prime} B^{\prime} C D$ ) is defined as the centroid of the quadrilateral (for proofs, see De Villiers, 1999; Yaglom, 1968).

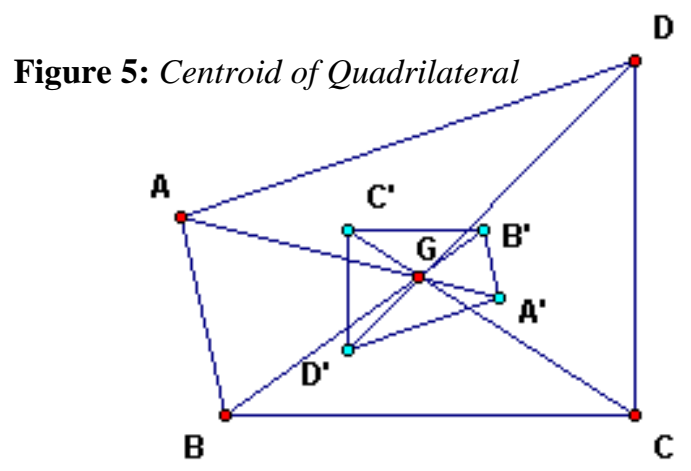


Another result we will need is the following. For a cyclic quadrilateral, the perpendiculars from the midpoints of the sides to the opposite sides (called the maltitudes) are concurrent as shown in Figure 6 (for a proof see De Villiers, 1999). This point of concurrency also coincides with the common point of intersection of the four nine-point circles of triangles $A B C, B C D, C D A$ and $D A B$, and is constructively defined as the nine-point (or Euler) centre of a cyclic quadrilateral by Yaglom (1968, p. 24).

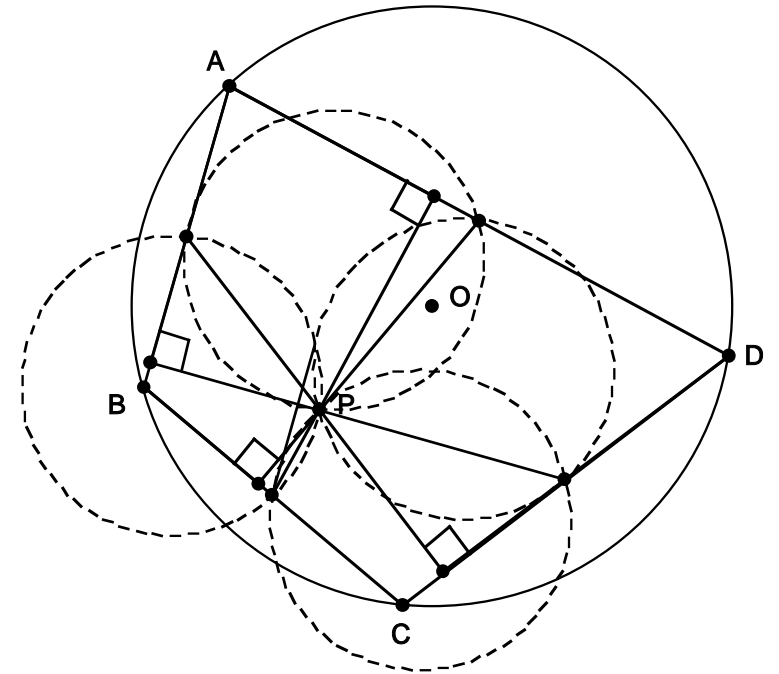

Figure 6: Nine-point centre of a cyclic quadrilateral

Then as shown in Figure 7, we have the interesting generalisation of the Euler line, namely, that the circumcentre $O$, nine-point centre $P$, and centroid $G$ of a cyclic quadrilateral are not only collinear, but also $O G=G P$ (for a proof, see De Villiers, 1999).
Maintaining the same relationship as for a triangle between the circumcentre $O$, nine-point centre $P$, and orthocentre $H$, we can now also constructively define the 'orthocentre' of a cyclic quadrilateral as a point $H$ so that $O P=P H$, and $O, P$, and $H$ are collinear.

Now similarly to the case for a triangle, note according to the given ratios between the points, that a dilation of $-1 / 3$ with centre $G$, maps $A B C D$ onto the centroid quadrilateral $A B^{\prime} C D^{\prime}$, and circumcentre $O$ to $O^{\prime}$. Hence, if $G O^{\prime}=x$, then $G O$ $=3 x$. But a dilation with a scale factor of 3 from centre $O$, maps $A B^{\prime} C D^{\prime}$ to $A^{\prime \prime} B^{\prime \prime} C^{\prime \prime} D^{\prime \prime}$, and $O^{\prime \prime}$ to $H$. Therefore, $H$ (which we defined as the orthocentre of a cyclic quadrilateral) is the circumcentre of $A " B=C{ }^{\prime \prime} D^{\prime \prime}$, and we have $\mathrm{OH}=3$ $\times O O^{\prime}=3 \times 4 x=12 x$ and $H G=9 x=3 G O$ (and from our definition of $H$ in terms of $P$ it follows that $P O=6 x$ and $P G=4 x=G O$ ).

On the basis of the analogy between the Euler and Nagel line of a triangle, and the above result for a cyclic quadrilateral, we can now constructively define the Nagel line for a quadrilateral circumscribed around a circle as follows (see Figure 7). For the circumscribed quadrilateral, a dilation of $-1 / 3$ with centre $G$, maps $A B C D$ onto the centroid quadrilateral $A B{ }^{\prime} C D^{\prime}$, and incentre $I$ to $I^{\prime}$, and because of the half-turn, $I, G$ and $I^{\prime}$ are collinear. Next apply a dilation with a scale factor of 3 from centre $I$, to map $A B^{\prime} C D^{\prime}$ to $A^{\prime \prime} B^{\prime \prime} C^{\prime} D^{\prime \prime}$, and $I^{\prime \prime}$ to the point $N$, which we now constructively

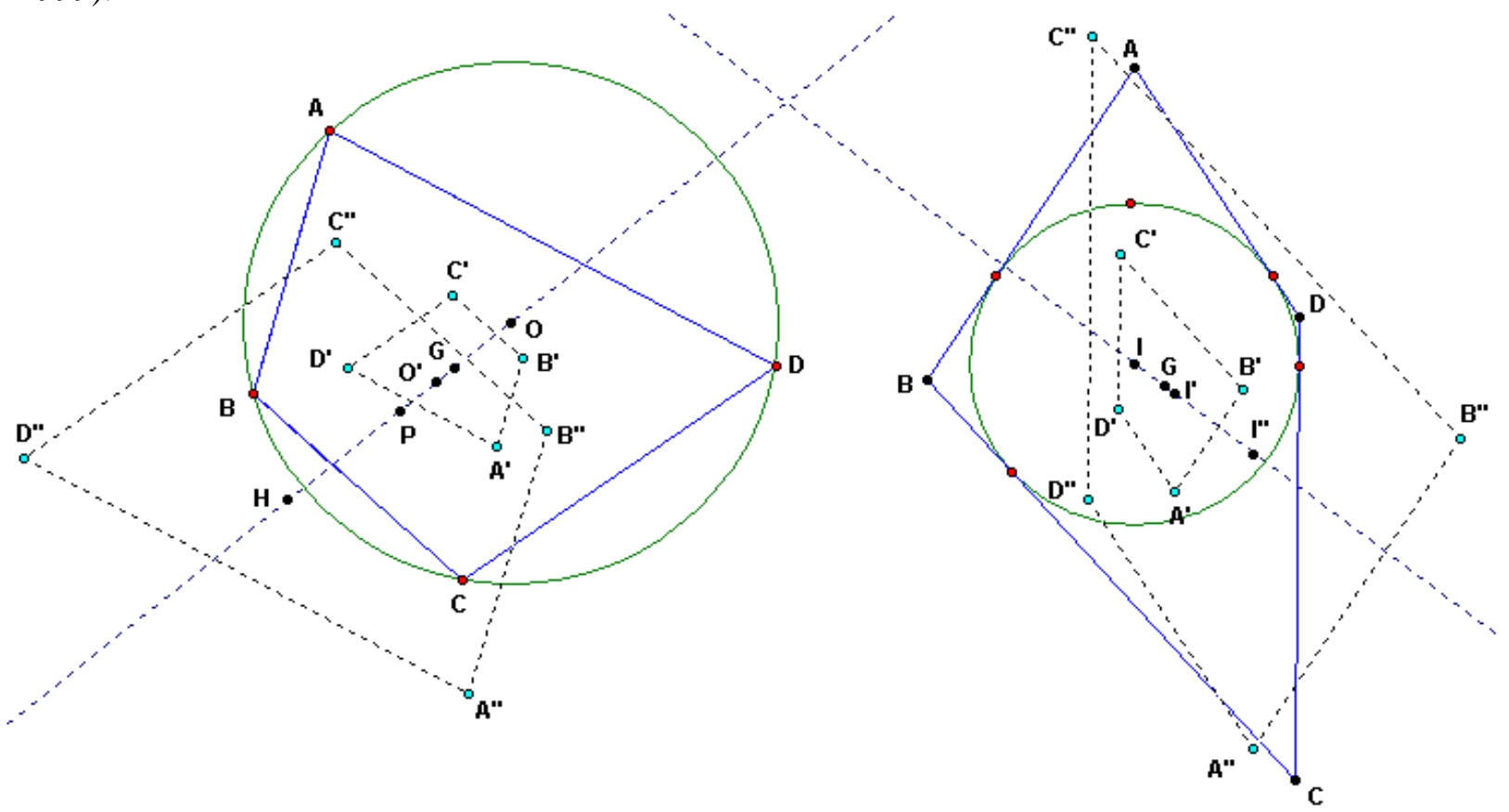

Figure 7: Euler line of cyclic quad and Nagel line of circum quad 
define as the Nagel point of a circumscribed quadrilateral. Then from the applied transformations we have similarly to the cyclic case that $N, G$ and $I$ are collinear, and $N G=3 G I$. If we analogously define the Spieker centre $S$ as the midpoint of $N I$, we also have $S G=G I$.

\section{Generalising the Euler and Nagel lines to cyclic and circumscribed polygons}

The generalisation of both results depend on the following general theorem for the centroid of any polygon, given and proved in De Villiers (1999) and Yaglom (1968): "Given a $n$-gon $A_{1} A_{2} A_{3} \ldots A_{n}$ $(n \geq 3) \ldots$, then the centroids of the (n-1)-gons, $A_{1} A_{2} A_{3} \ldots A_{n-1}, A_{2} A_{3} A_{4} \ldots A_{n}$, etc. that subdivide it, form a $n$-gon $A_{1}^{\prime} A_{2}^{\prime} A_{3}^{\prime} \ldots A_{n}^{\prime}$ similar to the original $n$-gon with a scale factor of $-\frac{1}{n-1}$, while the centre of similarity is the centroid of the original $n$-gon.”

Using this general result, we now constructively, and in general, define the orthocentre and Nagel point of a cyclic and circumscribed polygon, respectively, as the circumcentre and incentre of the $n$-gon $A_{1}^{\prime \prime} A_{2}^{\prime \prime} A_{3}^{\prime \prime} \ldots A_{n}^{\prime \prime}$ obtained from the dilation of the respective centroid polygon $A_{1}^{\prime} A_{2}^{\prime} A_{3}^{\prime} \ldots A_{n}^{\prime}$ with scale factor $n-1$.

As shown in Figure 8, if for example for the general Euler line we let $G O^{\prime}=x$, then

$G O=(n-1) x, O O^{\prime}=n x$ and

$H G=n(n-1) x-(n-1) x=(n-1)^{2} x$.

Hence, $\frac{H G}{G O}=\frac{(n-1)^{2} x}{(n-1) x}=n-1$.

It also follows that since $P$ is the midpoint of $\mathrm{OH}$ that $\mathrm{PG}=\frac{n(n-1) x}{2}-(n-1) x=(n-1) x\left(\frac{n}{2}-1\right)$, and $\frac{P G}{G O}=\frac{n}{2}-1$, etc. In exactly the same way, it follows for the general Nagel line that $\frac{N G}{G I}=n-1$ and $\frac{S G}{G O}=\frac{n}{2}-1$.

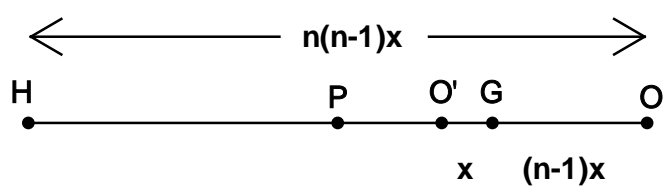

\section{Concluding comments}

Note that in the physical, real world context, the centroid given above is for point masses located at the vertices of cyclic and circumscribed polygons. Myakishev (2006) provides a completely different generalisation of the Nagel line of a circumscribed quadrilateral by considering instead the centroid of a 'perimeter' circumscribed quadrilateral (in other words, where all the weight is distributed along the boundary), and constructively defining a different Nagel point.

According to Benson (2007, p. 4), apart from its usefulness in discovering new knowledge, the ability to recognise analogies is also fundamental to problem solving as it "allows the solver to connect the familiar (a previously used method, strategy, or context) to the unfamiliar (a new problem)". Problem solving research has indeed revealed that expert problem solvers in mathematics and science engaged "in metaphorical processes as they constructed problem representations, they looked for analogies between the problem at hand and other familiar situations" (Silver, 1987, p. 45). An immediate research question that comes to mind is whether and how such reasoning can be developed and taught to novice learners.

Stefan Banach (1892-1945), the founder of modern functional analysis, characterises great mathematicians by their ability to go further, and even spotting analogies between analogies: "Good mathematicians see analogies. Great mathematicians see analogies between analogies." (Banach, n.d.)

Brown and Porter (n.d.) argue that one of the reasons for the current usefulness and importance of Category Theory, particularly in its application to scientific problems, is that it gives "an abstract mathematical setting for analogy and comparison, allowing an analysis of the process of abstracting and relating new concepts. This setting is one of the most important routes for the application of Mathematics to scientific problems.”

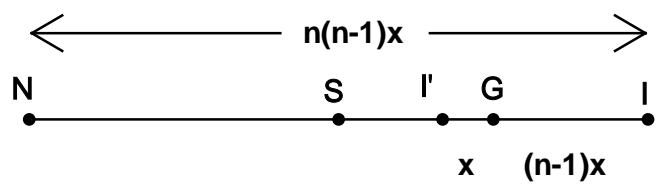

Figure 8: General Euler and Nagel lines 
It would appear that reasoning by analogy is a fundamental human ability and relates to our attempts to construct meaning and draw relationships between similar concepts or objects. From a very young age children are able to identify analogies, e.g. a lion is like a large cat, or a burrow for a rabbit is like a nest for a swallow, and as adults we often use analogies to communicate more effectively. Already in ancient Greek philosophy as well as the Bible there appear excellent analogies - the fables of Aesop and the parables of Jesus draw powerful analogies with the circumstances of people, and used to convey a moral or religious message. In traditional IQ and SAT-tests, simple analogies like "legs are to mammals as ... are to fish" have been frequently used as test-items to measure "intelligence", "academic potential” or "reasoning ability".

Use of analogy in the classroom by teachers can allow students to observe and use commonalities between different mathematical concepts, algorithms, representations, theorems, classifications, etc., thereby contributing to better integration of different components of mathematical knowledge. Most teachers probably use analogies such as 'solving inequalities is like solving equations', or 'balancing equations is like balancing a scale'. However, since analogies highlight commonalities, but not the essential differences, teachers ought to ensure that learners do not lose track of important underlying or fundamental distinctions.

In fact, the use of some popular analogies can be dangerous as pointed out by cognitive research over the last thirty years. For example, simply stating, 'doing algebra is just like doing arithmetic with letter symbols', in many ways seriously trivialises the abstract meaning of letter symbols representing "variables" or "generalised numbers" (Hart, 1981). Even worse, is the ubiquitous use of the false or misleading analogy that $2 x+3 x=5 x$ because 2 apples plus 3 apples is 5 apples. Sadly, some teachers often still say "we can't add $2 x+$ $3 y$ " because they are "unlike terms" and we can't add apples and oranges. This completely misleading use of letter symbols representing 'concrete objects' has been aptly called "fruitsalad” algebra.

Other analogies may help learners to remember results, e.g. a 'negative times a negative is positive since magnets of same polarity repel each other', but provides no explanation why the result is true. In such cases, they only serve the purpose of a mnemonic (memory aid device), but provide no meaningful integration with other mathematical concepts, and no different from rote learning of "rules without reasons".

Finding analogies between the properties of geometric figures on the plane and on the sphere (as well as major differences) can assist learners in developing a deeper understanding of the propositional nature of mathematics. More-over, according to Lenart (2004), learning comparatively about different types of geometries can help students develop a more tolerant view toward other people with opposing views, or of different social or cultural background, or when getting into another political, economical or technical environment.

Given the rich, multi-lingual and cultural diversity of South Africa, it seems a pity that very little research on analogical reasoning in mathematics seems to have been done locally. A study by Richland, Zur and Holyoak (2007) found that though mathematics teachers in Hong Kong and Japan compared to their USA counterparts used about the same number of analogies, they generally offered more cognitive support to explore the analogies more deeply and thoroughly. In particular, there was much more emphasis in the Asian countries on relational reasoning, and the use of mental and visual imagery. Taken altogether, these factors may well conceivably contribute to differences in performance in TIMMS achievements for these countries (Richland et al., 2007).

Of relevance to mathematics teachers and mathematics education researchers is a book edited by English (2004) reporting the results of a 3-year longitudinal study whose participants were children in Australia and the United States. In particular, the book seeks to understand the relationship between mathematical reasoning and children's natural tendency to create analogies. The book reports the results of empirical studies, as well as classroom discourse and case studies, which qualitatively analyse the role of discourse in the development and relationship between mathematical and analogical reasoning. 


\section{References}

Banach, J. (n.d.). Quote. Retrieved 4 December 2009 from http://en.wikipedia.org/wiki/Stefan_Banach

Benson, S. (2007). Problem Solving by Analogy/Problem Solving as Analogy. The Mathematics Educator, 17(2), 2-6. Retrieved 4 October 2008 from http://math.coe.uga.edu/TME/Issues/v17n2/v17n2 _Benson.pdf

Brown, R., \& Porter, T. (n.d.). Category Theory: An abstract setting for analogy and comparison. Retrieved on 21 December 2008 from www.informatics.bangor.ac.uk/public/math/resear ch/ftp/cathom/05_10.pdf

Coolidge, J. L. (1971). A Treatise on the circle and the sphere. Bronx, NY: Chelsea Publishing Company.

De Villiers, M. (1986a). Boolean algebra at school (Revised Edition). Stellenbosch: Research Unit for Mathematics Education at the University of Stellenbosch (RUMEUS).

De Villiers, M. D. (1986b). The role of axiomatization in mathematics and mathematics teaching. Stellenbosch: RUMEUS. Retrieved on 10 December 2009 from http://mzone.mweb.co.za/residents/profmd/axiom.pdf

De Villiers, M. (1999). Generalizations involving Maltitudes. International Journal of Mathematics Education, Science and Technology, 30(4), 541548.

De Villiers, M. (2000). A Fibonacci generalization and its dual. International Journal of Mathematics Education, Science and Technology, 31(3), 447477.

De Villiers, M. (2006). A generalization of the Spieker circle and Nagel line. Pythagoras, 63, 3037.

Department of Education (DoE). (2002). Revised National Curriculum Statement Grades R-9 (Schools), Mathematics. Pretoria: Department of Education.

English, L. (Ed.) (2004). Mathematical and analogical reasoning of young learners. New Jersey: Lawrence Erlbaum.
Hart, K. (1981). Children's understanding of mathematics: 11-16. London: John Murray.

Honsberger, R. (1995). Episodes in nineteenth \& twentieth century Euclidean Geometry. Washington, DC: The Mathematical Association of America.

Human, P. G. (1978). Wiskundige werkwyses in wiskunde-onderwys. Unpublished doctoral dissertation, Stellenbosch University, South Africa.

Freudenthal, H. (1973). Mathematics as an educational task. Dordrecht: Reidel.

Klein, F. (1924). Elementarmathematik vom höheren standpunkte aus (3rd ed.). Berlin: Springer.

Krygowska, A. Z. (1971). Treatment of the axiomatic method in class. In W. Servais \& T. Varga, Teaching school mathematics (pp. 124-150). London: Penguin-Unesco.

Lénárt, I. (2004). Why teach plane and spherical geometry simultaneously? AMESA KZN Mathematics Journal, 8(1), 39-48.

Myakishev, A. (2006). On two remarkable lines related to a quadrilateral. Forum Geometricorum, 6, 289-295.

Richland, L. E., Zur, O., \& Holyoak, K. J. (2007). Cognitive supports for analogies in the mathematics classroom. Education Forum, 316, 1128-1129.

Silver, E. (1987). Foundations of cognitive theory and research for mathematics problem solving. In A. Schoenfeld (Ed.), Cognitive science and mathematics education (pp. 33-60). New Jersey: Lawrence Erlbaum.

Toeplitz, O. (1963). The Calculus, a genetic approach. Chicago: University Press.

Weil, A. (1940). A letter to his sister on analogies in Mathematics. Retrieved 4 October 2008 from www.ams.org/notices/200503/fea-weil.pdf

Yaglom, I. M. (1968). Geometric transformations II. Washington, DC: The Mathematical Association of America. 